\title{
CREATIVE THINKING: COMPUTATIONAL TOOLS IMBUED WITH AI
}

\author{
R. E. Wendrich $\otimes$ \\ University of Twente, The Netherlands \\ $\square$ r.e.wendrich@utwente.nl
}

\section{Abstract}

This paper presents a test bed for AI technology on the integration of creative AI (CAI) with hybrid design tools (HDTs). The objective is to build and develop tools and programs for creative people (e.g. designers, engineers) to use, whereby the artificial intelligence (AI) software acts as a creative collaborator rather than a mere tool. The goal is to find a set of guiding principles, metaphors and ideas that inform the development of a CAI praxis imbued with computational support tools, new theories, experiments, and applications. Results and findings are presented of early-stage research.

Keywords: artificial intelligence (Al), design creativity, design tools, cyber-physical systems

\section{Introduction}

This paper presents the exploration and preliminary investigation of an approach to couple the natural human intelligence with the assistive potential of artificial intelligence (AI) through machine learning and deep learning networks in combination with blended environments and hybrid tools for design engineering processes (DEP). The act of creation (Koestler, 1964) often entails the dynamic process of creativity unleashed during design engineering processes (DEP). However, this may or may not lead to a creation of some sort or kind of artefact per se. The early stage(s) (phases) of the creative thinking process (sometimes called fuzzy frontend (FFE)), wherein thoughts and fuzzy notions are transformed and represented, often stem from the mind's eye (inner visions), metacognitive aspects, imagination, mental divisions and distractions. Devotion and intent are fused together to bring out ideas and fuzzy assumptions to manifest 'brain generated' content through the creative force and applied as elements in the creative act. An idea is essentially a creation. Whether an idea is creative, is judged based on its novelty and usefulness (Amabile and Pratt, 2016). The contribution is the experimentation, integration and testing of human-machine interaction (HMI) imbued with AI, i.e. Convolutional Neural Network $(\mathrm{CNN})$. The paper presents early-stage results and findings of data (i.e. input-output) generated during ideation and/or multiple design stages with the use of hybrid design tools and environment (HDTs, HDTE) (Wendrich, 2010, 2016) and by intertwining these data sets with creative AI (CAI) to support and assist during DEP.

\section{Creative and heuristic thinking}

Throughout DEP, problem-framing, FFE, and/or creative concept generation, it is widely recognized that designers and engineers find it hard to ignore obvious constraints, consequently ignore blind spots and/or impediments on their imagined iterative 'concepts,' before they have been fully created 
and/or developed. According to Crilly et al. (2008) the designer, like the consumer, is characterised by his or her experiences, beliefs, motivations, expectations, capabilities and culture. Mannheim (1939) stated, '...that the world is not given to us simply by our senses - we interpret it, through lenses based on culture, position, interests, and - ideologies.' The designer also has some anticipation of the eventual consumer, including some intentions for how that consumer should respond to the product (Figure 1 left).

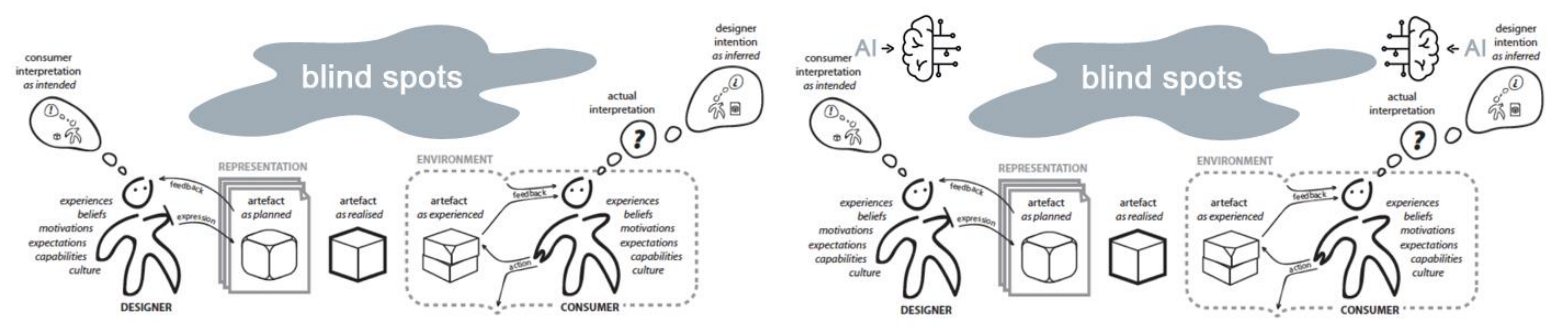

Figure 1. The friction between intention and inference: framed as apparent blind spots

Blind-spots are unexpected, unseen, unknown, unforeseen and/or ignored areas of knowledge or gaps in understanding and experience in the real-world context and within social-technological systems. They are a combination of low predictability and large impact once they become apparent (Wendrich and D'Cruz, 2011). These phenomena have direct implications on the human metacognitive aspects of creative process, intuition, imagination and creativity (Wendrich, 2010, 2016). Creative and heuristic thinking allows the creative act to initiate (ignites) with a complete and boundary less attitude towards inner and outer space, meaning that the current state-of-affair and context should have no bearing on the thought process or externalization of ideas. Amabile (1998) frames five components that affect creativity; encouragement at multiple levels, autonomy or freedom, resources, pressures (i.e. positive and negative) and impediments (e.g. conservatism, internal strife, too much design thinking). Therefore, deployment and integration of intelligent machines (e.g. thinking machines) could be beneficial, helpful and assistive during DEP to give and get valid information, seek out and provide directly observable data, create conditions for free and informed choice, facilitate awareness of values at stake in choice-architecture and decision making (Figure 1 right).

\subsection{Rational, irrational, arational}

According to Wendt (2017), the extend in which rational thinking is useful for designers and engineers, should be called into question. Mlodinov (2008) stated, '... that user behaviour is not only unpredictable, but also often irrational, and it is impossible to precisely know and control the circumstances and much is left to chance.' The machine progressively nudges towards new iterative steps or transformations to follow up, generate workflow that subsequently results in highly productive creative activity, playful interaction, rich and engaged creative processing. We concur with Dalcher (2006), that design is neither orderly nor linear; it implies a continuous and active search to resolve trade-offs and satisfying constraints. Colwell (2015) states, if designers/engineers only had to follow a set of directions, we wouldn't need designers/engineers; computers and robots can do that much. In the physical realm the use of tools shows a variety and diversity in use and outcome when used by a plethora of different users. This is often due to or a direct consequence of (intrinsic) skill set, context, experience, knowledge, under-standing and insight in tool use and its prospective usefulness. The idiosyncratic qualities and capacity will become visible directly and without any hidden surprise (Wendrich, 2010, 2015). Mannheim's (1939) way of putting it: "The ideas expressed by the subject are thus regarded as functions of his existence. This means that opinion, statements, propositions, and systems of ideas are not taken at their face value but are interpreted in the light of the life situation of the one who expresses them."

\subsection{Frames, metaphors and analogy}

Generative DEPs, are pretty much a 'mess.' Just try and make sense of the range of the terms floating around out there: from user-centered design, eco-design, design for the other $90 \%$, universal design, 
design thinking, sustainable design, interrogative design, task-centered design, reflective design, design for well-being, critical design, speculative design, towards speculative re-design...and innovation driven and so forth. Predominantly during the first phases of a DEP fostering creative thinking progress through a variety of pathways and constructs often aligned along symbolic cognitive (mental) representation modalities such as frames, metaphors and analogies in order to arrive at multitudes and generations of ideas. This points to cross-modality information transfer (Nóbrega et al., 2018), in which multiple signals (i.e. visual, oral, acoustic, olfactory, tangible) are transformed and expressed into symbolic visual representations. A frame in social theory consists of a schema of interpretation, which is a collection of anecdotes and stereotypes. According to Goffman (1974), people understand to be the organization of their experiences, they buttress, and perforce, selffulfilling. They develop a corpus of cautionary tales, games riddles, experiments, newsy stories, and other scenarios which elegantly confirm a frame-relevant view of the workings of the world. Designers and/or engineers construct (perhaps even fabrications) a set of mental filters through biological, emotional, economic and cultural influences. The choices they make are influenced by their creation of a frame. Framing can affect the outcome of a choice problem. The framing effect, one of the cognitive biases, describes that presenting the same option in different formats can alter people's decisions (Kahneman et al., 1982). Metaphors are used more frequently during the early, problem framing, stages of the design process and analogies are used later in the concept generation phase. Metaphors can be described as; 'a figurative expression which interprets a thing or action through an implied comparison with something else; a symbol', analogy as 'illustration of an idea by means of another familiar idea that is similar or parallel to it in some significant features'.

\section{Assisted creation and creativity}

In problem solving, the use of information-processing systems (i.e. thinking-machines, designmachines, teaching-machines, deviation amplifying inventing machines) that create and synthesize problem representations, manifest possible solve-for-solution searches selectively through rhizomes of intermediate situations, seeking the goal (target) situation and using heuristics to guide its search could be promising pathways. Following Maruyama (1963), that a machine that incorporates randomness, deviation-amplification and deviation-counteracting may be both efficient and flexible. It can search for all possibilities. It can try to amplify certain ideas in various directions. It can stay at a relevant idea (which may change from time to time during the invention) and bring back to it other ideas for synthesis (ibid.). 'The paths open to curiosity are many or even too many, they are never straight or predictable, and it takes different and unpredictable amounts of time to traverse them,' to paraphrase Nowotny (2010). Integration and implementation of artificial systems in conjunction build and develop tools and programs for the 'comprehensive designer' (Buckminster Fuller, 1969) to use for creative expression, idea- and conceptual generation (inputs) leading to new creative processes and outputs and replicas to make originals whereby the artificial intelligence (AI) software acts as a creative collaborator rather than a mere tool (Figure 2) and integrator of knowledge from for example associative network reasoning and representation (Figure 3) (phrasa.com). The research question and objective are to harmonize input-output (IO) of the analogue and digital realms (i.e. blended spaces, ubiquitous hybrid design tools (HDTs) and environments). In addition, the goal to improve individual and collaborative expression, creativity, representation, and communication imbued with creative artificial intelligence (CAI).
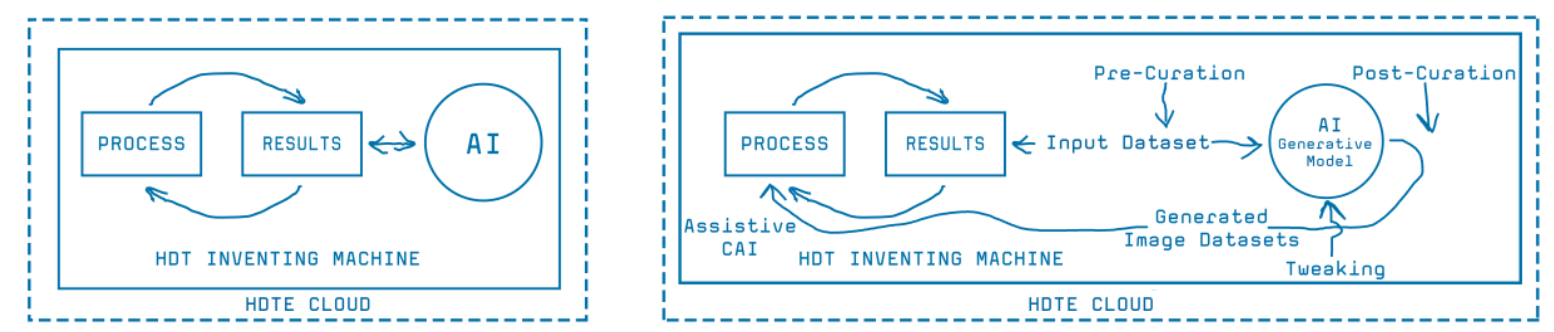

Figure 2. The inventing machine, environment and process 


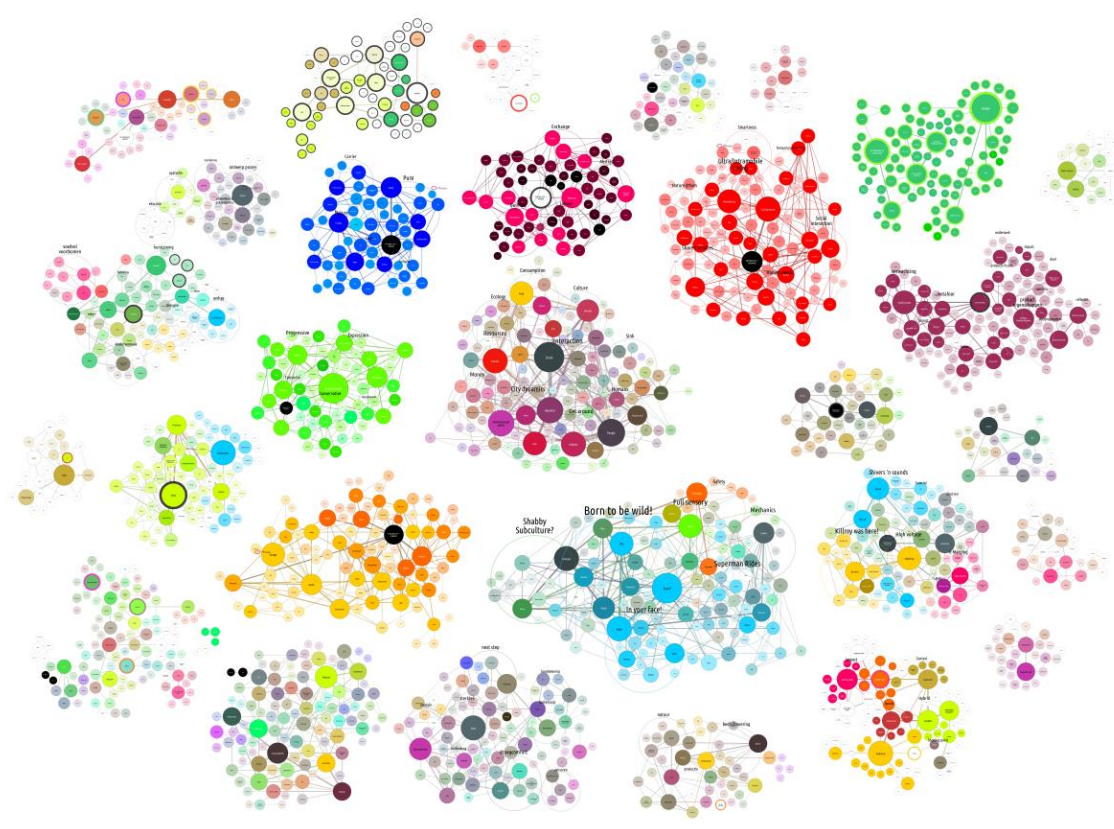

Figure 3. Associative network outcome and creative synthesis

\section{Generative creation and style transfer}

The exploration and inspiration were based on the creation of a convolutional neural network (CNN) that is capable of transferring style from one source (i.e. instance, stack, merge) and to incorporate it within a HDT and HDT Environment (HDTE) used for FFE during DEP (Brock et al., 2016; Dai et al., 2016), as shown in Figure 4.
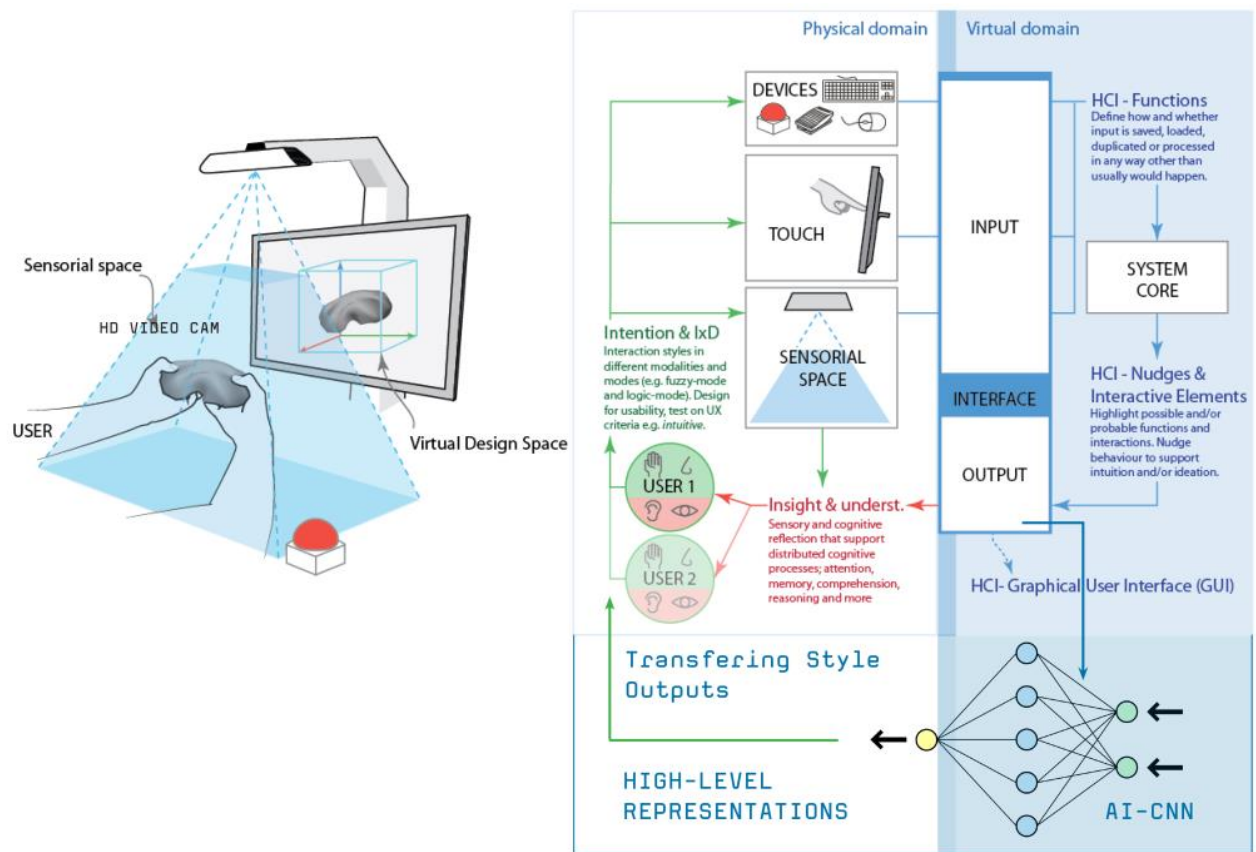

Figure 4. HDTE with AI-CNN integration

The initial testing focused on generation of new images with the use of two representational images (i.e. instances, merges, stacks) stemming from the HDT interaction process, as shown in Figure 5, Figure 6 and Figure 7. Deep Learning (DL) enables us to do things with algorithms that have never been done previously. Gatys et al. (2015) presented an approach to achieve this way of transferring style to one another. The early stages (i.e. fuzzy front-end) of any design engineering process (DEP) 
are often the most uncertain, whilst to obtain precise or exact information to generate requirements and/or feature lists is usually impossible to do from the onset (Koen et al., 2002; Robertson and Radcliffe, 2009; Vuletic et al., 2018). In such, the assistance and support by implementation of DL algorithms allow to take two separate neural representations of two given images, and then recombine them using a Deep Neural Network. In our study we deploy a convolutional neural network (CNN) which can be explained as an immense sequence of filters.
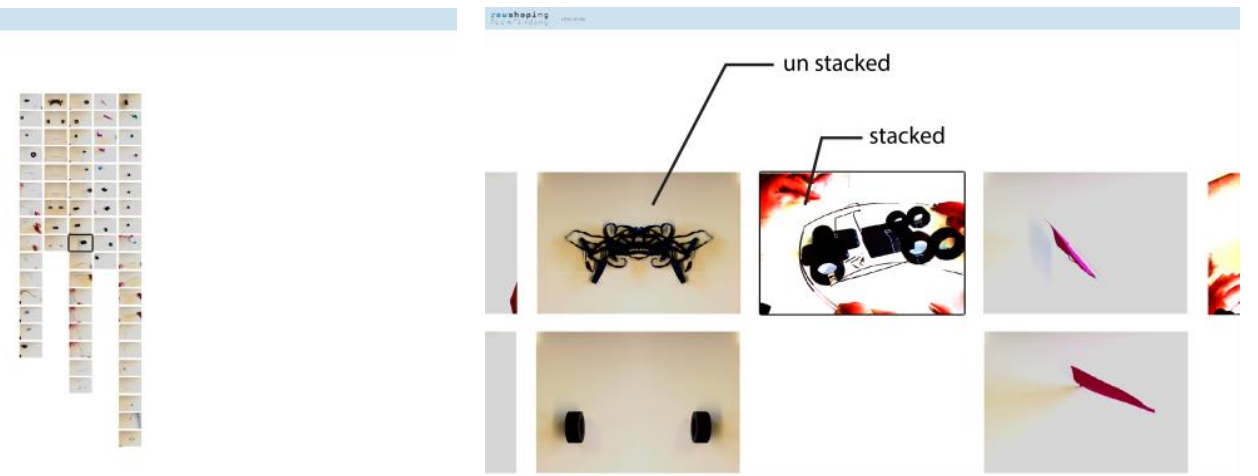

Figure 5. HDT representation iterative process flow (left), instances un- and stacked (right)
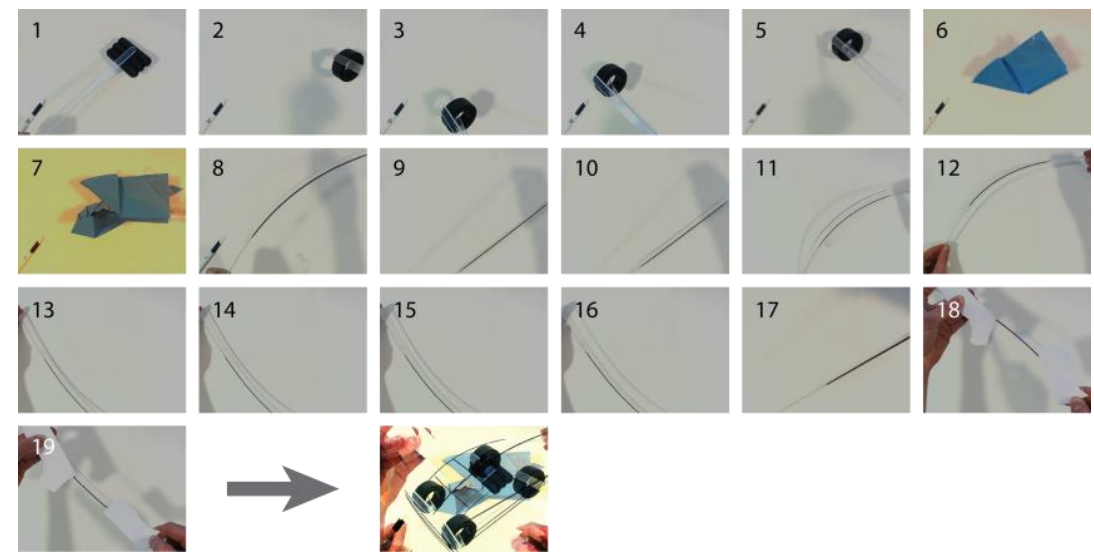

Figure 6. Typical iteration flow step 1 through 19 and result (stack) HDT (mid-bottom)

The output files of the HDT are layered instances (Figure 4) and end-results are either stacked or intermediate saved instances (in fact multiple contents resources are valid as inputs) being used as original inputs for the $\mathrm{CNN}$ to gain a newly generated image that serves as new input during the DEPFFE (Figure 7).
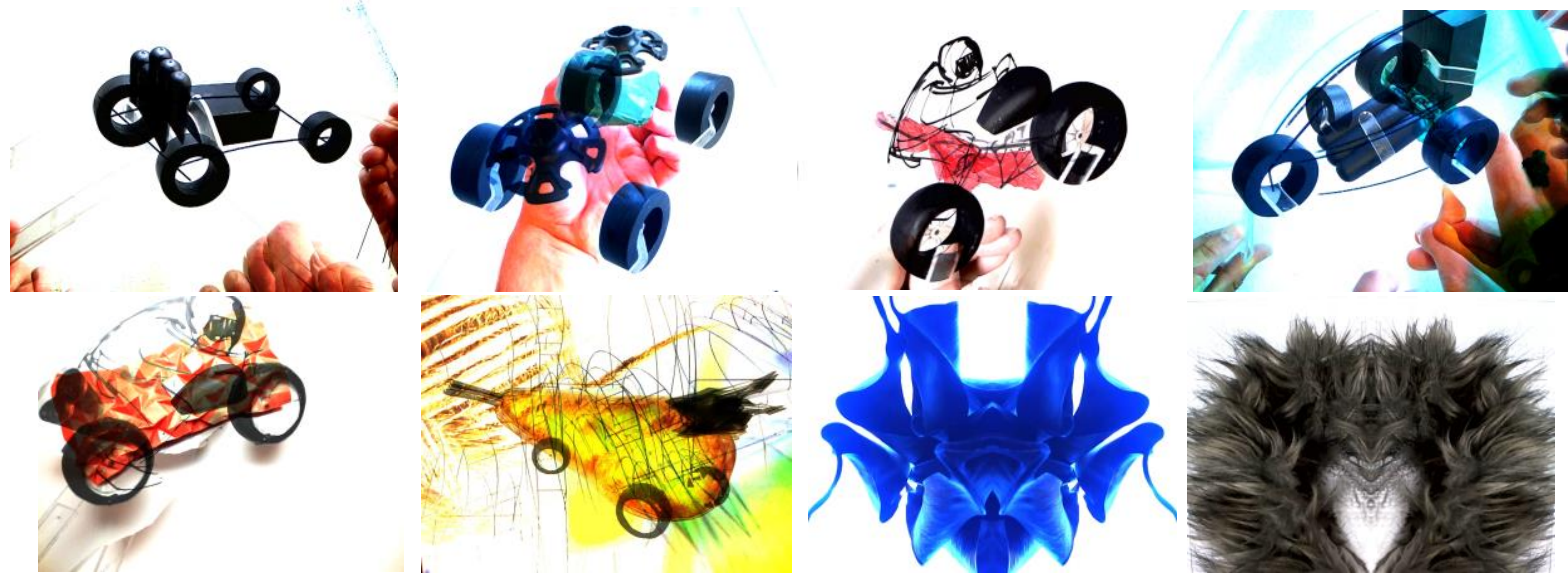

Figure 7. Generic and typical results (output) HDT process (i.e. instances, merges, stacks) 


\subsection{Initial testing HDT-CNN}

After multiple iterations and additional modifications were applied to the shape of the network, such as exploring the effects of a convolutional shape, which implies descending the number of neurons as the layers progress. The results became more promising, and once applied to a general image (stack) created by an HDT (Figure 8) the results were already starting to look a lot more like the original input.
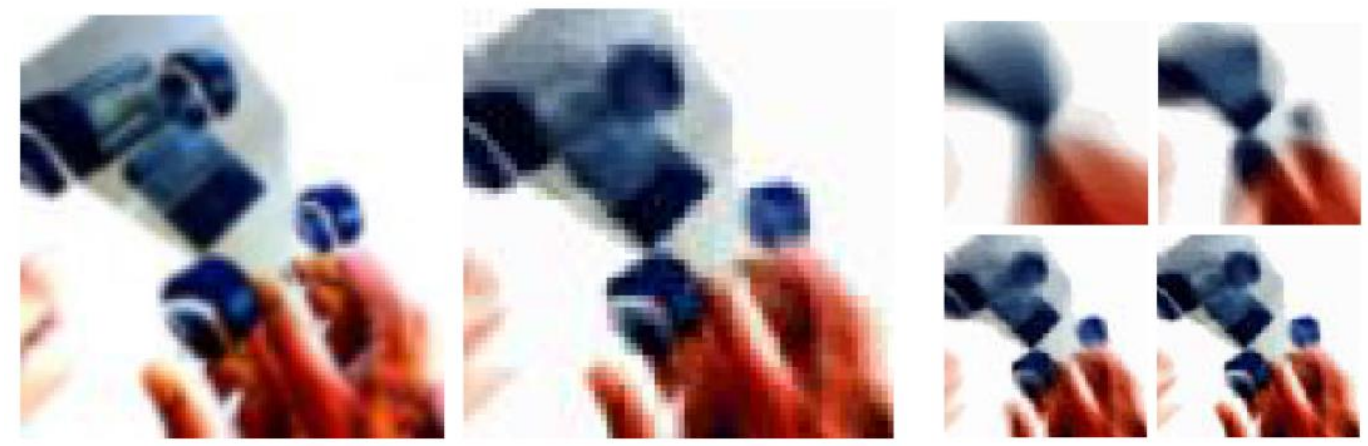

Figure 8. Left original image (stack), middle image-representation CNN, right excerpts generative process (low-resolution and low-level features)

\subsection{How the HDT-CNN works}

In order to integrate and embed CNN into the HDT(E) a short explanation on how it works is presented. A CNN could be explained as an immense sequence of filters. These filters are divided into smaller batches of following filters into the various hidden layers of the neural network (Lee, 2009).

A filter can be imagined as a 'small window' of just a few pixels that scans across an image. At the start of the network, these filters are sensitive to specific shapes. If this given shape is actively present in an image, they will provide a significant output to the next filters. These 'shapes,' for which a filter is sensitive, will from now on be referred to as features. At the start of the network, within the first layer, these filters are sensitive to rather small features, which can be as simple as a line or an edge. Within the next layers, the shapes for which these filters give a high output will become more involved with each layer as the filters start to combine themselves to more complex forms. As for illustrational purposes, the first layers will activate for some simple lines, which then in the following layers can be combined into an ear, the final layers are then able to reconstruct the face of a cat based on the combined lower-level features provided by the previous layers. As a rule of thumb, the further down you go in the network, the more accurate the representation of features will be in comparison to its original input: the input image is being reconstructed layer by layer through all from the edges that were detected in the first steps of the network, patched together by the subsequent layers. Given that the network is large enough, it should be able to reconstruct the original image with extreme accuracy, while also being aware of what shapes are present in the picture at hand, as shown in Figure 7 and Figure 10. CNN can recognize objects and /or identities with extreme accuracy, this is done by extending the network with a few extra classification layers (CL). These layers take the input that was reconstructed by the layers just before the CL and measure their 'output-strengths.' The CL then checks which neuron(-s) gave a strong output, and maps this to a classification mapping. This way each output is matched to an object and/or identity. If the output-strengths is high it is highly likely this object or character is present within the given image as input to the network. At present we will leave the final classification layers aside (not important for now). We focus in this trial on the network's ability to recognize and learn to identify edges and shapes that makes this networkarchitecture so useful for the HDT-CNN implementation and integration.

\subsection{Style representation and transferring style}

The essential trick that makes it possible in a Neural Network (NN) to learn how to recombine highlevel representations all the way up from the identified lower-level features in its other layers, is 
through the optimization function (i.e. cost function). This function allows the network to compare its output (i.e. predictive) with the actual goal (i.e. target). The optimization function compares how close the prediction of a network is to the target, and then predicts what small tweaks need to be made to the network to improve the prediction next time. This process of prediction, comparing and finally followed by tweaking is performed countless times until the network gives a prediction (an imagerepresentation) that is identical to the target (the original image). This process is also called gradient descent: it is the key that allows NN to learn from their mistakes, literally. Within the current context, this function can be very roughly simplified to:

representation quality = original image - output image

The compared images are being examined at the basis of their RGB values in every pixel (a perfect output image provide a blank canvas through this function). The goal of the network is to minimize this function. The lower the value of this function the better the NN is performing at recreating the original image. In such recreating a single image with the use of $\mathrm{NN}$ with this approach works rather well. When two images need to blend by transferring style to one another, such a simple function cannot be used anymore. Instead, another solution is provided by Gatys et al. (2015) in which two 'original' images, one for content representing the object, and one image to absorb the style, are considered as near perfect (raw) representation of the original, i.e. 'content representation.' In such, the NN recreates the original style image as a 'set of lower-level style representations.' The NN attempts to minimize the following equation (simplified):

$$
\begin{aligned}
& \text { total representation quality }= \\
& \text { content representation + the set of lower-level style representations }
\end{aligned}
$$

Instead of averaging the pictures, as a result, it adapts the higher-level features of the content image to be recast using the lower-level features of a given style representation. Therefore, the style will look and feel the same as the style-representation, while the objects displayed in the content-representation will still be recognizable as the higher-level features remain. Therefore, the function attempts to reach an optimum at which the lowest minimum possible will be achieved.

\subsection{Implementation and modification of CNN with $\mathrm{HDT}(\mathrm{E})$}

Adaptations will be implemented to the algorithm presented by Gatys et al. (2015). These changes aim to give the end-user more control over the algorithm's 'effectiveness' and to provide the result without having significant delays. By allowing the network to give output as its trains, the user can already start to see what the network will be aiming for given more time. The training process makes the style representation more useful over time as the creation-process is happening. It also provides the user with the opportunity to stop the training, should the style representation become too dominant to his or her liking. An additional modification to be implemented is the addition of controllable parameters, which modify the style transferring-process, which the user can tweak and experiment with. These parameters serve as knobs to fine-tune the perceived depth of the style transfer, the (dis) favouring of style over content and the ability to boost the rate of learning of the algorithm for faster ('rawer') results. TensorFlow (2015), an open source software library for high-performance numerical computing, was used extensively to construct, train and use the NN. TensorFlow (2015) is supported in multiple programming languages and can be used in a web-browser environment. Given that the HDT-LFDS can be connected to the internet and works in the browser, it can access pre-trained models and therefore leverage their capabilities without the need of training their own network or spending significant time on training their ML-models. Several test and iterations were made before we could apply the gained knowledge and insights to the HDT architecture and system. Additional modifications were applied to the shape of the network, such as exploring the effects of a convolutional shape, which implies descending the number of neurons as the layers progress. The results were more promising, and once applied to a general image created by an HDT-LFDS (i.e. instances, merges, stacks) as shown in Figure 7 and Figure 8, the results were already starting to look a lot more similar to the original input, as presented in Figure 9 and Figure 10. The results still leave plenty of room for improvement; for reference, the current accuracy of the representation is about 
$80 \%$. To speed the process up, we decided to stop experimenting with the effects of dimensionchanges on a network and opt for a pre-trained CNN instead; the VGG19 model. This model was also used as a baseline for the algorithm of Gatys et al. (2015). As this network achieved nearly identical replications of given input, there no need to further focus on this part. We included some tweaks within the CNN with the aim to give the user more control over the style transfer process. These options for adjustments were added over time and as follows:

- User sees the results of style transfer prior to having a fully trained network. Twofold benefit, less time consuming and possible termination (immediate, intermediate) of training for readjustment.

- User can modify and experiment what layers are being used for extracting content and style.

- User can select intensity and type of noise of original canvas; e.g. giving some 'starting noise' makes it easier for the network to find edges to nestle the style-reference.

- User can apply weights of the layers used for style-transferring. This allows the user to make the style express itself more aggressively when increased.

- User has control of the modification parameter for (dis-)favouring style over content. The loss-function evaluates and optimizes to have both the content-representation and the stylerepresentation present in the image.

\section{Style extraction and insertion for adoption in creative HD process}

We expanded on the work of Gatys et al. (2015) and propose a solution that can give HDTs a new way to expand what users can do with their images, intermediate results and/or creative outcome.
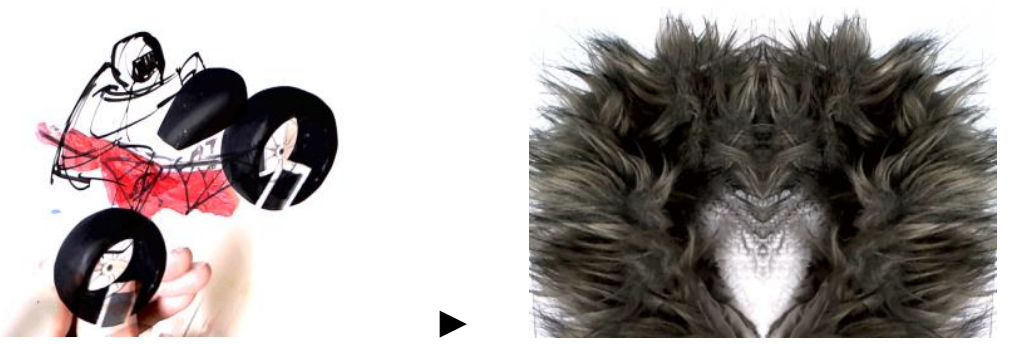

Figure 9. Style extraction (left-original image) and insertion (right-adoptive image)

The implemented solution makes use of an algorithm that can extract the style of one image and inserting it into another while maintaining the contents of the original image (Figure 10). Furthermore, currently we work on two suggestions (options) how these algorithms could be implemented within a HDTE. The first option would be to make the algorithm apply a layer-wise-adoption.
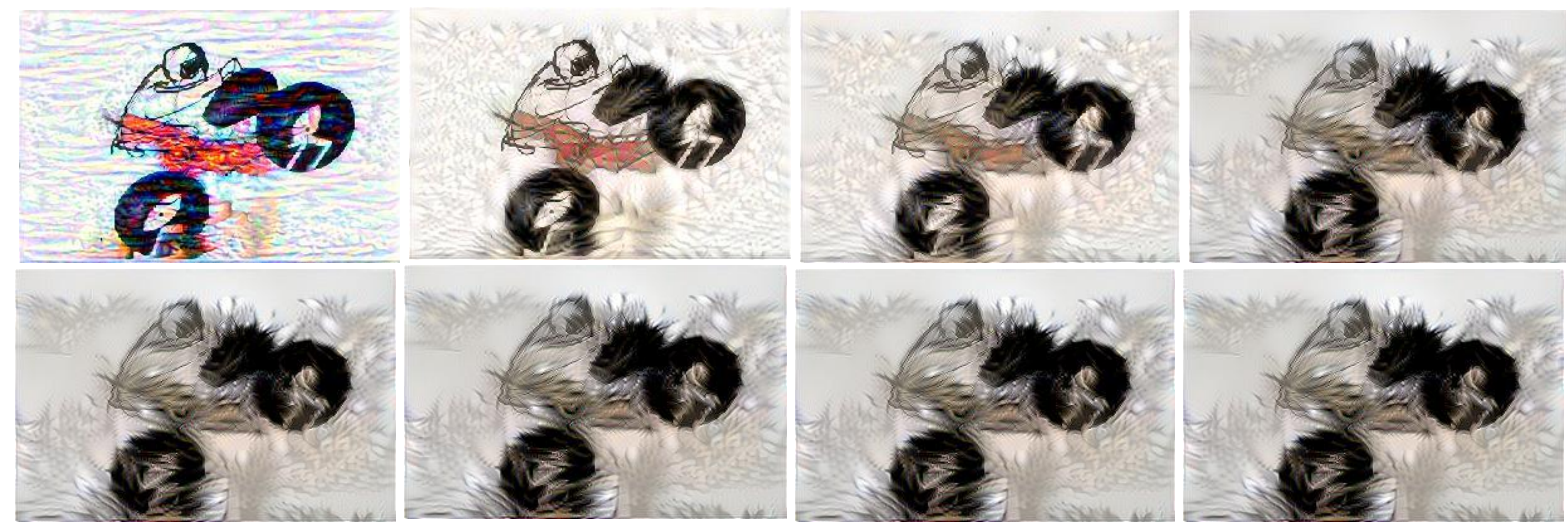

Figure 10. Image transformation through style-extraction in progress (samples only)

In fact, to paraphrase MacEachren (1995), abstract images can take on characteristics of real objects, and eventually come to be viewed as uncontested facts... The power of such master images to popularize 
theory is clear. In between frames or intermediate instances (as shown in Figure 10), the HDT can check what has changed in-between captures, and only affect a style of alteration to these 'new' pixels. The other option would be to give the algorithm the capabilities to detect textures or different surfaces in an image. Then, the algorithm could apply specific modifications to each identified texture/surface. However, some trade-offs will need to be made before implementation. However, in the long run, possible 'a rather short sprint,' more computing power will become available, and the algorithms that can be used to apply these modifications to pictures will become even more useful. Therefore, it is interesting to consider possible areas for further development: possibilities to further expand the effectiveness and control, so to say, over the way in which style-transfer could be applied to images. Yet, the algorithm is only capable of applying one style to an image. By improving the algorithm to be able to use multiple sources at once, would significantly enhance its potential.

\section{Conclusion}

Creativity is a key ability of individual(s), has supposedly an intentional activity (process), the process takes (in general) place in or within a specific context (environment) and brings about generation of ideas, artefacts, product(s) and/or objects (tangible or intangible). The outcome should be valuable, meaningful, useful, spark off novel thoughts, perspectives and venues for further deliberation, iteration and communication. The application of style transfer can be successfully integrated and embodied within the HDT-LFDS in multiple ways and different scenarios. Goldschmidt (2015) states that potential visual design and engineering stimuli are ubiquitous if one is attentive enough to be able to capture and harness them to serve as sources of inspiration in the DEP. The current status of the HDTCAI system is the flexibility, the desired speed of the CNN is still a challenge. It must have undergone extensive training before being use functionally fluid and assistive in (near) real-time. Using a decent GPU (in our case NVIDIA GeForce GTX970), it takes the original algorithm roughly a few hours of training time before its usable. Furthermore, the result is something that must be seen at the end of the tunnel; the network does not provide outputs of intermediate results. For the use-case of these experiments, having such a delay in seeing one's results is just not practical, neither would the preparation of style representations in advance be. Therefore, a faster alternative need to be explored: by allowing the network (cloud) to give output as it trains, the user can already start to see what the network will be aiming for given more time. Furthermore, it should be noted that this training-phase needs to be done for each new style-representation that is introduced to the system. To provide the image used for as style-representation on the spot: it does not need to be trained within the model a priori. However, once the training has been done, the model can be stored for later use, or even distributed to other systems. In doing so, a collection of trained representations can be maintained to mitigate for this drawback. Further research and experimentation are underway to explore and investigate other possibilities of CNN in HDT-LFDS and HDTE. The creative human capability (e.g. thinking, reflection), competence and capacity (e.g. skills), to playfully collaborate in design processing bringing imagination and inspiration to the design process at hand, coincides with the intuitive natural human ability to interact, communicate and challenge life force against creative force in a direct and/or indirect way. Creativity imbued with CAI is an ability to discover new ideas, define problems, discover blind spots and address challenges to solve for solutions.

\section{Acknowledgement}

The author likes to thank Max Meijer for his work and contribution.

\section{References}

Amabile, T.M. (1998), How to kill creativity (Vol. 87). Boston, MA: Harvard Business School Publishing.

Amabile, T.M. and Pratt, M.G. (2016), "The dynamic componential model of creativity and innovation in organizations: Making progress, making meaning", Research in Organizational Behaviour, Vol. 36, pp. 157-183.

Brock, A. et al. (2016), "Generative and discriminative voxel modelling with convolutional neural networks", arXiv preprint arXiv: 1608.04236.

Colwell, R. (2015), "Our computer systems are not good enough", In Energy Efficient Electronic Systems (E3S), 2015 Fourth Berkeley Symposium on IEEE, pp. 1-1. 
Crilly, N., Maier, A. and Clarkson, P.J. (2008), "Representing artefacts as media: Modelling the relationship between designer intent and consumer experience", International Journal of Design, Vol. 2 No. 3, pp. 15-27.

Dai, J., Lu, Y. and Wu, Y.N. (2016), "Generative modeling of convolutional neural networks", Statistics and Its Interface, Vol. 9 No. 4, pp. 485-496. https://doi.org/10.4310/sii.2016.v9.n4.a8

Dalcher, D. (2006), "Consilience for universal design: the emergence of a third culture", Universal Access in the Information Society, Vol. 5 No. 3, pp. 253-268.

Fuller, R.B. (1969), "Ideas and integrities: A spontaneous autobiographical disclosure”, Estate of R. Buckm. Fuller.

Gatys, L.A., Ecker, A.S. and Bethge, M. (2015), “A neural algorithm of artistic style”, arXiv: 1508.06576.

Goffman, E. (1974), Frame analysis: An essay on the organization of experience, Harvard University Press.

Goldschmidt, G. (2015), "Ubiquitous serendipity: Potential visual design stimuli are everywhere”, In Studying visual and spatial reasoning for design creativity, pp. 205-214. Springer Netherlands.

Kahneman, D., Slovic, P. and Tversky, A. (1982), Judgment and Uncertainty: Heuristic and Biases.

Koen, P.A. et al. (2002), Fuzzy front end: Effective methods, tools, and techniques, Wiley, New York, NY.

Koestler, A. (1964), The act of creation.

Lee, H. et al. (2009), "Convolutional deep belief networks for scalable unsupervised learning of hierarchical representations", In Proc. of the 26th Ann. Int. Conf. on M.L. ACM.

Mannheim, K. (1939), "Ideology and utopia: An introduction to the sociology of knowledge", Brace and Company.

Maruyama, M. (1963). The second cybernetics: Deviation-amplifying mutual casual processes.

MacEachren, A.M. (1995, 2004), "How maps work: representation, visualization, and design", Guilford Press. American Scientist, Vol. 51 No. 2, pp. 164-179.

Mlodinow, L. (2008), The Drunkard's Walk: How Randomness Affects Our Lives.

Nóbrega, V.A., Miyagawa, S. and Lesure, C.L. (2018), Cross-Modality Information Transfer: A Hypothesis about the Relationship among Prehistoric Cave Paintings, Symbolic Thinking, and the Emergence of Language.

Nowotny, H. (2010), Insatiable curiosity: innovation in a fragile future, MIT Press.

Robertson, B. and Radcliffe, D. (2009), "Impact of CAD tools on creative problem solving in engineering design", Computer-Aided Design, Vol. 41 No. 3, pp. 136-146.

TensorFlow (2015). https://www.tensorflow.org/, Last Accessed: November 11, 2019

Vuletic, T. et al. (2018), "The challenges in computer supported conceptual engineering design", Computers in Industry, Vol. 95, pp. 22-37.

Wendrich, R.E. (2010), "Raw Shaping Form Finding: Tacit Tangible CAD”, Computer-Aided Design and Applications, Vol. 7 No. 4, pp. 505-531. https://doi.org/10.3722/cadaps.2010.505-531

Wendrich, R.E. (2015), "Integrated Creativity and Play Environments in Design and Engineering Processes", Volume 1B: 35th Computers and Information in Engineering Conference. https://doi.org/10.1115/detc201547214

Wendrich, R.E. and D’Çruz, M. (2011) in Wendrich (2016), "Hybrid design tools for conceptual design and design engineering processes: bridging the design gap: towards an intuitive design tool".

Wendt, T. (2017), “Arational design”, In: Relating Systems Thinking and Design Symposium (RSD), 13-15 Oct 2016, Toronto, Canada. Available at http://openresearch.ocadu.ca/id/eprint/1935/ 